\title{
Commentary
}

\section{Ethnicity, race or nation? Census classifications as barriers to the measurement of mixedness in Australia}

\author{
Catriona Stevens* The University of Western Australia \\ Farida Fozdar The University of Western Australia \\ * Corresponding author. Email: catriona.stevens@uwa.edu.au. Address: School of Social Sciences, \\ The University of Western Australia, 35 Stirling Highway, Perth, WA 6009, Australia.
}

Paper received 1 March 2021; accepted 25 April 2021; published 31 May 2021

\section{Introduction}

It is an unfortunate truth that all systems of ethnic and racial classification are in some sense 'wrong', with each failing to adequately capture the complexity of a given population. This potentially perpetuates and legitimises structures of (dis)advantage based in culture or visible difference. Regardless of approach taken, such classification systems will likely confuse, obscure and/or offend at least some members. But given Australia's diversity, its history as a settler colony dispossessing and numerically overwhelming its Indigenous peoples, its decades as 'White Australia', its more recent welcoming of migrants from a range of ethnic, racial, religious and national backgrounds, and its growing 'mixed' population, such systems are vital for determining who 'we' are and for mapping these changes and their wider correlates. Our particular interest is in the (in)ability to identify the extent, composition and rate of mixed populations in Australia. But effective classification and measurement of the ethnic and racial identities of census respondents will also support the accurate targeting and outcomes of multicultural service provision, and help scholars, policy makers, and ethnic communities understand demographic change over time.

The current Australian census classification system is a great improvement on earlier approaches that were informed solely by the logics of biological race in the service of a desired White nation (Fozdar and Stevens 2020). Yet the Australian Standard Classification of Cultural and Ethnic Groups (ASCCEG), the statistical standard used by the Australian Bureau of Statistics (ABS) for the collection, aggregation and dissemination of data relating to the cultural and ethnic diversity of the Australian population, is, like many other classificatory systems, still strange and inconsistent. The current approach is limited and anachronistic. This is not only an issue for social scientists seeking to understand these changes and related socioeconomic patterns. The census also sends a message, signalling the types of information deemed valid and worth collecting, and the sorts of personal characteristics seen as culturally important.

In early census schedules, a race question was included in order to monitor the size of the nonEuropean population. For example, in 1911 the categories employed were: Asiatic, African, American, 
Polynesian, and Indefinite, with each category subdivided into 'Full blood' and 'Half-caste'. In this rendering, mixedness was only relevant insofar as it involved European and non-European 'blood'. Following the introduction of anti-discrimination legislation in 1975, the steady retreat from the earlier racist logic of the census categories led to a complete loss of race-based categories. The only exception is Indigeneity, with a question asking whether the respondent is "of Aboriginal or Torres Strait Islander origin".

Since 1986, ethnicity has replaced race as the key official measure of communities of descent, with the ASCCEG introduced as the statistical standard in 2000. However, the categories employed remain limited, and in some cases are downright bizarre, for example, including explicitly racial categories alongside generic national identifiers that mask significant ethnic and racial diversity (discussed shortly). While recognising the need for consistency over time, this commentary proposes some changes to permit expanded ways of measuring ethnic and particularly racial mixedness while minimising disruption to longitudinal studies.

\section{Limitations of ASCCEG classifications and aggregation mechanisms}

We argue the ASCCEG classification categories require reform due, in part, to a range of issues including: the current inconsistency and confusion in terms of the logic of categorisation; the increasing diversity of the population and its desire to acknowledge these backgrounds; and the growing levels of mixedness in Australia. With regard to the latter, it is important to have the capacity to measure the mixed population due to its transformative potential (King-O'Riain et al. 2014; Parker and Song 2001; Meyer and Fozdar 2016). This includes not only mapping the changing 'face' of Australia, but also mixedness's ability to erode entrenched spatial segregation (Tindale and Klocker 2014, 2017), both resulting from and generating a 'contact' effect - lower levels of prejudice and improved cross cultural relations (Pettigrew and Tropp 2006). However, considerable analytical contortionism is necessary to derive 'mixedness' from the current classification system. This is partly because of the lack of a 'race' based question. This is the focus of our discussion.

For the 2021 Census, the ASCCEG system is used to code responses to the ancestry question (household paper form question 22). It is applied to aggregate base-level four-digit ancestries, the most fine-grained level of analysis, into two-digit narrow groups and one-digit broad groups. These broad groups typically equate to continent of ancestry. This commentary primarily relates to question 22 and the related statistical standard, but also makes reference to the following 2021 Census topics: main language other than English used at home (question 20); country of birth (question 16); and Indigenous status (question 11). We are interested in these topics in relation to the ability to classify people of mixed backgrounds, and also in relation to race or visible difference.

One of the main points we wish to emphasise is that there are significant inconsistencies in how the ASCCEG defines 'cultural and ethnic groups', with confusion about national, racial and ethnic categorization leading to often functionally meaningless classifications. The base level 4-digit categories variously describe subnational ethnic groups (for example, "5206 Sundanese", an Austronesian ethnic group from western Java, and the second largest ethnic group in Indonesia), ethno-national categories (such as "6901 Japanese"), national categories without ethnic or racial specificity (such as "8203 Brazilian", collapsing people of White, Mixed, Black, Asian and Indigenous 
backgrounds), and explicitly racial/panethnic categories (for example "8105 Hispanic [N American]", a category specifically for those from North America of Latino or Spanish ancestry). Of this range of categorisations, national categories without ethnic or racial specificity are by far the most common, reinforcing a supposed alignment of race/culture/ethnicity with nation; and perpetuating the pervasive methodological nationalism that has been critiqued for its theoretical and practical inadequacy and anachronism (Beck 2007).

The logics of the ASCCEG are perhaps most strange when considering the Americas. Ancestry options for people of North America are essentially racial, while those from other countries are not. North American options include "8101 African American", "8105 Hispanic [North American]" and "8106 Native North American Indian" alongside the racially more ambiguous (although presumably White) "8102 American" and "8103 Canadian". These base level ancestries reflect some of the racial categories from the US Census (Herman 2020) and appear to have simply been imported wholesale from that. Yet other classificatory systems from other countries, many of which are inflected with legacies of similarly racist and racialized histories (Aspinall and Rocha 2020), are not. For example, explicitly racial categories from the British census (e.g., Asian British [meaning primarily people of South Asian ancestries]; Black Caribbean; Black African) are missing, with those identifying with British ancestries assumed to be White. Thus, there is apparently little consistency, and an unclear logic, to the categories available for use, leading to a differential ability among analysts to clearly identify sub-national ethnic and racial backgrounds of respondents. This makes more detailed analysis of populations difficult. It also limits respondents in their ability to identify in ways they may prefer.

There are further troubling inconsistencies produced through the ways that categories are aggregated in the ASCCEG's hierarchical structure. Base level categories are aggregated on the basis of two classification criteria: geographic proximity of ancestral origins, and 'similar social and cultural characteristics' (ABS 2019). This is, however, a blunt tool, and in practice aggregation at both the broad and narrow levels conflates groups with different ethnic/racial backgrounds, or conversely, separates groups with similar backgrounds. As well as being logically inconsistent, both of these practices create barriers to measuring and analysing racial mixedness in the Australian population.

For example, "9201 Afrikaner", "9202 Angolan" and "9203 Eritrean" are the first three categories in the narrow group "92 Southern and East African". Geographic, social and cultural similarity, not to mention racial, is not evident here. An analysis of census data at the one- or two-digit level reveals high rates of mixedness between North-West Europeans and Southern and East Africans, yet a closer investigation would show that this rarely entails the racial mix that one might assume. Similarly, although North American base-level ancestries are explicitly racial, when aggregated to the broad level, this obscures visible difference and visible mixedness.

Meanwhile, other ancestries are separated into different narrow and broad aggregated groups for reasons that are unclear whether on grounds of geography, ethnicity or language. Two examples from Europe and Africa illustrate just how illogical some outcomes from the ASCCEG classification criteria may be. France and Spain are geographically proximate, sharing a border, and the countries feature many 'similar social and cultural characteristics' including, for example, speaking Romance languages and a history of adherence to Roman Catholicism. Yet "3106 Spanish" is placed in broad 
group "3 Southern and Eastern European", while "2305 French" aggregates to broad group "2 NorthWest European". This appears to reflect the legacy of historical othering of Southern Europeans, and implicit layered racism within the charter group.

ASCCEG explanatory notes acknowledge the problems of including "4201 Jewish" in broad group "4 North African and Middle Eastern", with community consultation failing to determine a better approach (ABS 2019). Yet there are other confusing aspects to this broad group that merit further attention. For example, narrow group " 49 Other North African and Middle Eastern" includes both "4904 Iranian" and "4913 Nubian". Peoples of the Sudan, meanwhile, are grouped together, notwithstanding the deep ethnic and cultural conflicts that have shaped and reshaped the national borders in this region, resulting in the strange situation where " 4305 South Sudanese" aggregates to broad group "4 North African and Middle Eastern" while people identifying with ancestries "9203 Eritrean" or "9204 Ethiopian" are considered part of broad group "9 Sub-Saharan African". And of course "9215 South African", which includes White, Black, Mixed and Asian people who have migrated from a country of 59 million, are also part of this group, together with, among others, "9235 Madi", a small ethnic group of about 400,000 Sudanic-speaking people living across Sudan and Uganda. Neither geographic, nor cultural, nor racial logics seem to apply.

Finally, and perhaps of greatest significance to our interest in mixedness in Australia, is that question 22 in the 2021 Census unduly obscures the multiple ancestral identities that are increasingly prevalent. Recognising Australia's growing diversity, the ASCCEG notes that "Many people will relate to more than one cultural or ethnic group and will give a multiple response to a question on ancestry... Data collection models should be developed to capture multiple responses to enhance usefulness of the output" (ABS 2019). However, in practice the census currently only permits two responses to the ancestry question. This is inadequate as Australians increasingly identify with many different cultural backgrounds (Fozdar and Stevens 2020; Fozdar and Volet 2016). As comparisons, respondents to the Canadian census are instructed to '[s]pecify as many origins as applicable' for the ancestry question and indicate all that apply for the ethnicity question (Kwan-Lafond and Winterstein 2020 pp. 80-82), while census respondents in Britain are invited to write in 'Any other Mixed/multiple ethnic background' in an open field (Aspinall 2020). These are far more flexible options that allow more accurate identification, honouring respondents' desire to acknowledge a range of backgrounds, and offering researchers the ability to track growing complexity.

\section{Alternative measures of ethnic and racial difference}

We propose that the ancestry question in the Australian census be amended to accommodate multiple responses, perhaps four to six options, rather than two, providing useful data on the kinds of mixedness appearing in the population, while also supporting respondent engagement with the Census through the official validation of diverse forms of identity and mixedness. While the ANCP1 (first stated ancestry) and ANCP2 (second stated ancestry) are important data outputs to preserve for longitudinal analyses, the inclusion of further ancestries should not impact the validity of these data.

We also propose further consultations to explore the merits of a population group question to better understand visible difference and visible mixedness in Australia. Such understandings are highly 
limited when relying on the country of birth, ancestry and Indigenous status data currently collected (Fozdar and Stevens 2020). There are precedents for this. In addition to an ancestry question very similar to that used in Australia, Statistics Canada includes a further 'visible minority' question that closely approximates a race question, namely "Is the person White / Chinese / South Asian / Black / Filipino / Latin American / Southeast Asian / Arab / West Asian / Korean / Japanese?" (Kwan-Laford and Winterstein 2020). Respondents are invited to tick all that apply, allowing for those who identify with multiple backgrounds to include these. The results of these data are not perfect: Arab, Latin American and West Asian, for example, are not considered 'visible minorities'. However, this question does offer a range of self-identifications that could provide an indication of the current 'racial' make-up of Australia, at least more clearly than can currently be inferred from ancestry responses aggregated at the broad and narrow level groupings.

We acknowledge that the inclusion of racial terminology may be controversial, given Australia's history, but point out that racial categories already exist, in the form of Indigenous classifications. Current practice, which frequently mixes racial, ethnic, national, and even geographical categories, is inconsistent and unhelpful. While we would not suggest that the ASCCEG and ancestry question be abandoned, due to the need to be able to make longitudinal comparisons, we do suggest some refinement and a new 'population group' question is required. Miri Song (2018) has argued that racial classification remains vital for academics and policy makers to understand racial difference and mixing. Official avoidance of the term in favour of ethnic identification, and in Australia's case, identification that confuses national, ethnic and racial categories, actually creates problems, as it makes it difficult to differentiate between different kinds of ethnic and racial experiences. It also makes it impossible to confidently draw conclusions about inter-ethnic and inter-racial unions. The current options thus invisibilise, or at best confuse, the dimensions on which differences of outcomes may rest.

\section{Discussion}

We acknowledge that ethnicity and race questions are difficult, given their potential to alienate, reference historical injustices, invisibilise, and to ignore specificities - as stated at the outset of this commentary - whatever choices are made will always be somehow 'wrong'. However, we argue more choice is better than less, and that respondents are capable of engaging with the range of options to self-identify in ways that are meaningful to them. We reiterate that the census, as the official tool of the nation for data collection, and the categories it uses, sends a message, signalling the types of information seen as valid and valuable, with implications for the imagining of the nationstate and its peoples, and its orientation to diversity and inclusion.

We have raised a number of questions above - why are the ASCCEG categories so arbitrary and imprecise, apparently importing US racial classifications for some people, and collapsing racial and ethnic categories into national options for others? Although only adopted in 2000, the ASCCEG appears embedded in the logics of an earlier era. As such, it is oriented towards the past, and may not currently be able to accommodate the complexities of future populations. We further have queried: What is the point of allowing people four-digit specific options if these are then so poorly aggregated, erasing that specificity? Is it realistic to allow people only two ancestry options, and what 
information is being lost as a result? How can mixedness be determined, with any degree of confidence, when the classifications are often at best loose and at worst arbitrary, and sometimes race-based, and sometimes not?

In summary, we recommend small, possibly incremental, changes that will:

- reduce inconsistencies in the ASCCEG, through the removal of classifications based on the racial logics of North America; and

- permit more than two ancestral identifications and hence data collection that reflects the changing demographic realities of (what we think is) our uniquely diverse Australian population.

We also recommend exploration of a race/population group/visibility question.

Likely benefits include:

- a more precise and accurate estimation of the racial diversity of Australia (often proportion of migrants is pointed to as evidence of Australia's racial and cultural diversity, but this tells us little about region of origin and cultural or racial background);

- the opportunity to map the changing profile of race in Australia;

- the opportunity to capture racial heritage of second and further generation migrants; and

- making race visible as a dimension of social life in Australia. This would allow local, state and federal governments to develop targeted interventions and services where needed, for ethnic communities to better estimate their numbers and geographic spread, and for businesses to target communities where appropriate.

Of course, any changes would require research to understand how they might be interpreted by the Australian community.

Overall, the issues raised are related to our interest in mixedness, and its extent, patterning and growth in Australia. This is important for the reasons raised above, as it potentially signals a movement towards a more diverse, less segregated and more tolerant society. But there are wider reasons for a review and revision of the ways the census approaches ethnic and racial diversity. Social scientists need to be confident about the logic of the classification system in order to make predictions and policy recommendations. And Australians need to feel their identities are appropriately acknowledged through official channels such as the census. This is vital for the development of an inclusive and just society.

\section{References}

ABS (2019) Australian Standard Classification of Cultural and Ethnic Groups (ASCCEG), 2019. https://www.abs.gov.au/ausstats/abs@.nsf/mf/1249.0. Accessed on 14 February 2021.

Aspinall P (2020) The Path to Official Recognition of 'Mixedness' in the United Kingdom. In: Rocha Z L and Aspinal P J (eds) The Palgrave International Handbook of Mixed Racial and Ethnic Classification. London: Palgrave Macmillan; 229-248. https://doi.org/10.1007/978-3-030-22874-3 12 
Aspinall P and Rocha Z (2020) Introduction: North and South America. In: Rocha Z L and Aspinal PJ (eds) The Palgrave International Handbook of Mixed Racial and Ethnic Classification. London: Palgrave Macmillan; 49-74. https://doi.org/10.1007/978-3-030-22874-3 3

Beck U (2007) The cosmopolitan condition: Why methodological nationalism fails. Theory, Culture \& Society 24(7-8): 286-290. https://doi.org/10.1177/02632764070240072505

Fozdar F and Stevens C (2020) Measuring mixedness in Australia. In: Rocha Z L and Aspinal P J (eds) The Palgrave International Handbook of Mixed Racial and Ethnic Classification. London: Palgrave Macmillan; 605-628. https://doi.org/10.1007/978-3-030-22874-3 32

Fozdar F and Volet S (2016) Cultural self-identification and orientations to cross-cultural mixing on an Australian university campus. Journal of Intercultural Studies 37(1): 51-68. https://doi.org/10.1080/07256868.2015.1119674

Herman M (2020) Methods of Measuring Multiracial Americans. In: Rocha Z L and Aspinal P J (eds) The Palgrave International Handbook of Mixed Racial and Ethnic Classification. London: Palgrave Macmillan; 95-112. https://doi.org/10.1007/978-3-030-22874-3 5

King-O'Riain R, Small S, Mahtani M, Song M and Spickard P (eds) (2014) Global Mixed Race. New York: New York University Press.

Kwan-Lafond, D and Winterstein, S (2020) The Canadian Census and mixed race: Tracking mixed race through ancestry, visible minority status, and metis population groups in Canada. In: Rocha Z L and Aspinal P J (eds) The Palgrave International Handbook of Mixed Racial and Ethnic Classification. London: Palgrave Macmillan; 75-94. https://doi.org/10.1007/978-3-030-22874-3 4

Meyer M and Fozdar F (2016) "A mini United Nations": Being mixed in multicultural Australia. In: McGavin $\mathrm{K}$ and Fozdar F (eds) Mixed Race Identities in Australia, New Zealand and the Pacific Islands. New York: Routledge; 49-66.

Parker D and Song M (eds) (2001) Rethinking 'Mixed Race'. Pluto Press.

Pettigrew T F and Tropp L R (2006) A meta-analytic test of intergroup contact theory. Journal of Personality and Social Psychology 90(5): 751-783. https://doi.org/10.1037/0022-3514.90.5.751

Song M (2018) Why we still need to talk about race. Ethnic and Racial Studies 41(6): 1131-1145. https://doi.org/10.1080/01419870.2018.1410200

Tindale A and Klocker N (2017) Mapping the multiple geographies of mixed-ethnicity couples in Australia. Australian Geographer 48(4): 473-495. https://doi.org/10.1080/00049182.2017.1353584

Tindale A, Klocker N and Gibson C (2014) Inter-ethnic partnerships: remaking urban ethnic diversity. Urban Policy and Research 32(4): 393-415. https://doi.org/10.1080/08111146.2014.883967 